\title{
Intrinsic Brain Connectivity in Fibromyalgia Is Associated With Chronic Pain Intensity
}

\author{
Vitaly Napadow, ${ }^{1}$ Lauren LaCount, ${ }^{2}$ Kyungmo Park, ${ }^{3}$ Sawsan As-Sanie, ${ }^{4}$ \\ Daniel J. Clauw, ${ }^{4}$ and Richard E. Harris ${ }^{4}$
}

Objective. Fibromyalgia (FM) is considered to be the prototypical central chronic pain syndrome and is associated with widespread pain that fluctuates spontaneously. Multiple studies have demonstrated altered brain activity in these patients. The objective of this study was to investigate the degree of connectivity between multiple brain networks in patients with FM, as well as how activity in these networks correlates with the level of spontaneous pain.

Methods. Resting-state functional magnetic resonance imaging (FMRI) data from 18 patients with FM and 18 age-matched healthy control subjects were analyzed using dual-regression independent components analysis, which is a data-driven approach for the iden-

The content of this article is solely the responsibility of the authors and does not necessarily represent the official views of our funding agencies.

Supported by the NIH (National Center for Complementary and Alternative Medicine grants K01-A002166 and R01-AT-004714 [to Dr. Napadow], F05-AT-003770 [to Dr. Park], and P01-AT-002048, K01-AT-01111 [to Dr. Harris], and National Center for Research Resources grants P41-RR-14075 and GCRC M01-RR-01066), the Mental Illness and Neuroscience Discovery Institute, and Pfizer Inc. Dr. Park's work also was supported by the Institute of Information Technology Advancement, Korea (IITA-2008 [C1090-0801-0002]). Dr. Harris' work also was supported by the US Department of Defense (Army grant DAMD-W81XWH-07-2-0050); he is recipient of a Dana Foundation Award in Brain and Immuno-Imaging.

${ }^{1}$ Vitaly Napadow, PhD: Massachusetts General Hospital, Charlestown, and Logan College of Chiropractic, Chesterfield, Missouri; ${ }^{2}$ Lauren LaCount, BS: Massachusetts General Hospital, Charlestown; ${ }^{3}$ Kyungmo Park, PhD: Kyunghee University, Yongin, Korea; ${ }^{4}$ Sawsan As-Sanie, MD, MPH, Daniel J. Clauw, MD, Richard E. Harris, PhD: University of Michigan, Ann Arbor.

Dr. Clauw has received consulting fees, speaking fees, and/or honoraria from Pfizer, Forest, Cypress Biosciences, Pierre Fabre, $\mathrm{UCB}$, and AstraZeneca (less than $\$ 10,000$ each). Dr. Harris has received consulting fees and honoraria from Pfizer (less than $\$ 10,000$ ) as well as research grant funding from Pfizer.

Address correspondence and reprint requests to Vitaly $\mathrm{Na}$ padow, $\mathrm{PhD}$, Martinos Center for Biomedical Imaging, Department of Radiology, Massachusetts General Hospital, 149 Thirteenth Street \#2301, Charlestown, MA 02129. E-mail: vitaly@nmr.mgh. harvard.edu.

Submitted for publication December 2, 2009; accepted in revised form March 30, 2010. tification of independent brain networks. Intrinsic, or resting-state, connectivity was evaluated in multiple brain networks: the default mode network (DMN), the executive attention network (EAN), and the medial visual network (MVN), with the MVN serving as a negative control. Spontaneous pain levels were also analyzed for covariance with intrinsic connectivity.

Results. Patients with FM had greater connectivity within the DMN and right EAN (corrected $P\left[\boldsymbol{P}_{\text {corr }}\right]$ $<0.05$ versus controls), and greater connectivity between the DMN and the insular cortex, which is a brain region known to process evoked pain. Furthermore, greater intensity of spontaneous pain at the time of the FMRI scan correlated with greater intrinsic connectivity between the insula and both the DMN and right EAN $\left(P_{\text {corr }}<0.05\right)$.

Conclusion. These findings indicate that resting brain activity within multiple networks is associated with spontaneous clinical pain in patients with FM. These findings may also have broader implications for how subjective experiences such as pain arise from a complex interplay among multiple brain networks.

Chronic pain disorders cause significant disability and dysfunction in patients and are particularly troublesome for both researchers and clinicians. Since pain is inherently a subjective sensation, clinicians and researchers must rely on the patient's self-report of pain severity. As such, the identification of objective markers that could simultaneously validate chronic pain symptoms and be used in elucidating underlying pathologic processes would be of significant benefit. Recent neuroimaging studies have focused on identifying neural correlates of chronic pain in human patients $(1,2)$. However, this approach has been notoriously problematic, because chronic pain is difficult to elicit in a controlled manner, since it arises spontaneously and can fluctuate in magnitude (3).

Fibromyalgia (FM) is considered to be the pro- 
totypical central, or "functional," chronic pain syndrome (4) and is associated with widespread pain. FM is also characterized by increased sensitivity to noxious and non-noxious stimuli (i.e., hyperalgesia and allodynia, respectively) and augmented brain responses to experimental painful stimuli $(5,6)$. Although their findings are informative, these latter studies, which have assessed thresholds of evoked experimental pain on brain imaging, provide little information regarding the underlying constituents of endogenous clinical pain. In fact, measures of experimental pain only show modest correlations with self-reported clinical pain in patients with FM (7).

Resting-state functional-connectivity magnetic resonance imaging (MRI) is a recent adaptation of functional MRI (FMRI) that may be promising for the assessment of associations of spontaneous functional pain with specific brain network activity (see Table 1 for key terminology). This method examines intrinsic connectivity, which is defined as ongoing neural and metabolic activity that occurs in the resting basal state. This intrinsic, or resting, state of the brain actually demands the bulk of brain-energy metabolism, in contrast to the relatively small fraction $(<5 \%)$ of energy metabolism demanded by stimulus-evoked activity (8). Although the role of intrinsic brain connectivity has not been definitively resolved, it may be important for maintenance of synaptic connectivity, such as the magnitude and extent of neuronal transmission between brain regions. Intrinsic connectivity may also involve information transfer between disparate brain regions, comprising known primary sensory, executive, and associative networks (9).

FMRI investigations of brain regions showing correlated activity in the resting state, referred to as intrinsic connectivity networks (ICNs), are conducted with an MRI scanner while the patient is in a resting state. The data derived from these investigations can then be analyzed with independent components analysis (ICA), which is a data-driven method of isolating independent brain networks that have temporally correlated FMRI findings on time-series scans. These brain networks are thought to be connected synaptically, since the FMRI signal between brain areas in these networks is correlated over time. Specifically, correlations of the ICN signal on FMRI follow known structural monosynaptic and polysynaptic pathways $(10,11)$, which is likely a reflection of neurophysiologically meaningful activity (12).

Although ICNs have been implicated as traits in several patient populations, the relationship of ICNs to state-dependent processes, such as chronic pain perception, remains largely unexplored. If intrinsic brain con-
Table 1. Glossary of relevant terms in the analysis of brain connectivity by FMRI in patients with FM*

\begin{tabular}{|c|c|}
\hline $\mathrm{DMN}$ & $\begin{array}{l}\text { This brain network is a constellation of } \\
\text { brain regions thought to be engaged } \\
\text { in self-referential thinking. This } \\
\text { network is "deactivated" during } \\
\text { various externally focused task } \\
\text { conditions-i.e., it is more active at } \\
\text { rest. Anatomically, it includes the } \\
\text { inferior parietal lobule, the posterior } \\
\text { cingulate cortex and precuneus, areas } \\
\text { of the medial frontal gyri, the } \\
\text { hippocampal formation, and lateral } \\
\text { temporal cortex. }\end{array}$ \\
\hline Dual-regression ICA & $\begin{array}{l}\text { This technique to analyze resting FMRI } \\
\text { data allows for the estimation of } \\
\text { resting-state, or intrinsic, functional } \\
\text { connectivity networks. FMRI data } \\
\text { from each subject are combined and } \\
\text { evaluated with ICA. These group } \\
\text { ICNs are then used to find individual } \\
\text { ICNs specific to each subject, which } \\
\text { are then used for within- and } \\
\text { between-subject analyses. }\end{array}$ \\
\hline EAN & $\begin{array}{l}\text { This brain network is involved with } \\
\text { cognitive processing of working } \\
\text { memory and attention. Anatomically, } \\
\text { it comprises the frontal and parietal } \\
\text { lobe regions, including the } \\
\text { dorsolateral prefrontal cortex } \\
\text { (roughly, the frontal eye fields) and } \\
\text { posterior parietal regions overlapping } \\
\text { the superior parietal lobule and } \\
\text { intraparietal sulcus. }\end{array}$ \\
\hline ICNs & $\begin{array}{l}\text { Networks of different brain regions that } \\
\text { are connected to each other in a } \\
\text { resting basal (as opposed to stimulus- } \\
\text { evoked) state. }\end{array}$ \\
\hline MVN & $\begin{array}{l}\text { This brain network includes primary } \\
\text { visual processing areas (VI) on both } \\
\text { hemispheres. Anatomically, it } \\
\text { includes regions along the calcarine } \\
\text { sulcus and lingual gyrus. }\end{array}$ \\
\hline $\begin{array}{l}\text { Spontaneous clinical } \\
\text { pain }\end{array}$ & $\begin{array}{l}\text { In FM, clinical pain fluctuates } \\
\text { spontaneously over time. In the } \\
\text { present study, spontaneous pain was } \\
\text { self-reported by patients with FM at } \\
\text { the time of the FMRI scan, and the } \\
\text { data were used to guide statistical } \\
\text { analysis. }\end{array}$ \\
\hline State-specific & $\begin{array}{l}\text { Pertaining to the state of mind that the } \\
\text { subject is in during the FMRI brain } \\
\text { scan. }\end{array}$ \\
\hline Synaptic connectivity & $\begin{array}{l}\text { Brain regions connected to one another } \\
\text { through at least one synapse between } \\
\text { two neurons. Information is } \\
\text { exchanged between these regions. }\end{array}$ \\
\hline
\end{tabular}

* FMRI $=$ functional magnetic resonance imaging; $\mathrm{FM}=$ fibromyalgia; $\mathrm{DMN}=$ default mode network; ICA = independent components analysis; ICNs = intrinsic connectivity networks; EAN = executive attention network; $\mathrm{MVN}=$ medial visual network.

nectivity were found to be correlated with the chronic pain state, it might lay the groundwork for the identification of much-needed objective biomarkers of clinical pain perception and open up several interesting avenues 
for research. Interestingly, the time scale of spontaneous pain fluctuation (in seconds to minutes [13]) is on the same order as the low-frequency intrinsic connectivity of the ICNs, providing an additional rationale for investigating the association between intrinsic brain connectivity and chronic pain.

Several ICNs have been identified in healthy subjects (14), while 2 important networks related to cognition and potential clinical pain are the default mode network (DMN) and executive attention network (EAN). The DMN $(9,15)$ is a constellation of brain regions thought to be engaged in self-referential thinking that are "deactivated" during various externally focused task conditions. Pain is known to influence both the DMN response and cognitive capacity. Whereas acute experimental pain induces DMN deactivation in healthy subjects (16), chronic back pain is associated with mitigated DMN deactivation during visual attention tasks (17). The frontoparietal EAN is a brain network that appears to be involved with cognitive processing of working memory and attention $(18,19)$. Patients with FM are known to experience concomitant cognitive deficits, which affect both working memory and attention processing (20), providing a rationale for the evaluation of intrinsic connectivity in the EAN.

Given that 1) the DMN response may be disrupted during a state of chronic pain, 2) cognitive deficits specific to working memory and attention are common in patients with FM (20), and 3) accumulating evidence suggests that intrinsic brain connectivity may be state-dependent $(21,22)$, we hypothesized that intrinsic DMN and EAN connectivity would be altered in patients with FM, and that these alterations would be associated with the level of spontaneous clinical pain. In order to strengthen the specificity of our findings, we also examined intrinsic connectivity in the medial visual network (MVN) as a negative control, since dysfunction in this primary sensory network has not been noted in patients with FM.

\section{PATIENTS AND METHODS}

Subjects and data collection. Data were collected from 36 female subjects in 2 separate groups, comprising 18 patients with FM (mean \pm SD age $38.9 \pm 10.8$ years) and 18 agematched healthy control subjects (mean \pm SD age $36.1 \pm 15.3$ years; $P=0.53$ versus patients with $\mathrm{FM}$ ). All participants gave their written informed consent, and the University of Michigan Institutional Review Board approved all study protocols.

All patients in this study were diagnosed as having FM according to the American College of Rheumatology (ACR) criteria for FM (23), with a disease duration of at least 1 year, and had reported the continued presence of pain for more than $50 \%$ of each day, were willing to limit the introduction of any new medications or treatment strategies for control of their FM symptoms during the study, were older than age 18 years and under age 75 years, were female, were right-handed, and were capable of giving their written informed consent. Patients with FM were excluded if they were currently taking or had a history of taking opioid or narcotic analgesics, had a history of substance abuse, had a concurrent autoimmune or inflammatory disease that causes pain, such as rheumatoid arthritis, systemic lupus erythematosus, or inflammatory bowel disease, were concurrently participating in other therapeutic trials, were pregnant or currently a nursing mother, had a severe psychiatric illness (e.g., current schizophrenia, major depression with suicidal ideation, or substance abuse within the past 2 years), or currently had major depression, the latter of which was assessed with either the Hospital Anxiety and Depression Scale (HADS) $(\mathrm{n}=8)$ or the Center for Epidemiologic Studies Depression Scale (CES-D) $(\mathrm{n}=10)(24,25)$. Categorization of patients with FM according to a low versus high level of depression was achieved by defining a high level as $\geq 8$ symptoms on the HADS and $\geq 16$ on the CES-D.

All healthy control subjects were between the ages of 18 and 75 years, were female, were capable of giving their written informed consent, were right-handed, and were willing to complete all study procedures. Exclusion criteria for healthy control subjects were having met the ACR criteria for FM, having any chronic medical illness, including a psychiatric disorder (e.g., psychosis, schizophrenia, or delusional disorder), and current pregnancy.

Six minutes of resting-state FMRI data were collected in the first functional scan run in the session. We used a spiral in-out gradient-echo $\mathrm{T} 2 *$-weighted $\mathrm{BOLD}$ pulse sequence (repetition time [TR]/echo time [TE] 2,000/30 msec, 180 volumes, 43 anterior commissure-posterior commissurealigned slices, voxel size $3.13 \times 3.13 \times 4.0 \mathrm{~mm}$ ) that was run on a 3T Signa EXCITE scanner (General Electric), equipped with an 8-channel head coil. Subjects were instructed to close their eyes and to rest comfortably during the functional scan, without moving or falling asleep. Structural data were also collected using a spoiled gradient-recalled acquisition in the steady state pulse sequence (TE $5.5 \mathrm{msec}$, TR $14 \mathrm{msec}$, inversion time $300 \mathrm{msec}$, flip angle $20^{\circ}$, number of excitations $=1,124$ contiguous axial slices, voxel size $1 \times 1 \times 1.5$ $\mathrm{mm}$ ). Prior to undergoing the scan, subjects were asked to rate the intensity of their FM pain on a visual analog scale of $0-10$, where 0 is equivalent to "no pain present" and 10 is equivalent to "the worst pain they could imagine."

Physiologic data were collected at the same time as the FMRI scan, because cardiorespiratory fluctuations are known to influence FMRI estimation of intrinsic connectivity within several brain networks $(26,27)$. Cardiac data were acquired using an infrared pulse oximeter (General Electric) attached to the right middle finger. Respiratory volume data were acquired using an MR-compatible belt (General Electric), which was placed around the subject's rib cage.

Analysis of FMRI data. Analyses of the FMRI data were performed using the validated software package FSL (available from the FMRIB Software Library at www.fmrib.ox.ac.uk/fsl). Data were corrected for motion artifact, compensating for any head movements using an FSL linear (affine) transformation (FSL-MCFLIRT) procedure. Extraction of functional data from the brain scan was performed using the FSL brain extraction tool (FSL-BET). Func- 
tional data were smoothed using a Gaussian kernel of 5-mm full width at half maximum. High-pass temporal filtering $(\mathrm{f}=$ $0.006 \mathrm{~Hz}$ ) was also performed, since we wanted to remove very low frequency scanner-drift artifacts, and the ICNs in our patients showed peak power at higher frequencies $(\sim 0.03 \mathrm{~Hz})$ $(28,29)$.

Analyses of the within- and between-subject restingstate FMRI data were performed using ICA with the FSL Multivariate Exploratory Linear Optimized Decomposition into Independent Components (FSL-MELODIC) tool and a previously validated dual-regression approach (30). This technique allows for voxel-wise comparisons of resting-state functional connectivity by, first, temporally concatenating restingstate FMRI data from all subjects, followed by backreconstructing the group ICNs for individual subjects, yielding data that can then be used for within-subject and betweensubject group difference maps. This technique has been found to have moderate-to-high test-retest reliability in previous studies (31)

Functional data were first projected to standard Montreal Neurological Institute space using structural/functional linear (affine) coregistration (FSL-FLIRT) and nonlinear structural/template coregistration using the FMRIB Nonlinear Image Registration Tool (FNIRT). These BOLD functional data (180 volumes for each subject) were then concatenated in time across all subjects, creating a single 4-dimensional (4-D) data set. We then applied probabilistic ICA (with the FSLMELODIC tool) to identify global, distinct (independent) patterns of functional connectivity in the entire subject population (covering both patients with FM and healthy control subjects). We limited the number of independent components (ICs) in this step to 25 . This was done to minimize IC split ting into subcomponents, as recommended by Filippini et al (30).

From this pool of $25 \mathrm{ICs}$, ICNs of interest were selected using our goodness-of-fit method (21), with previously defined templates from Beckmann et al (14). Briefly, the best-fit component was selected by calculating the average $\mathrm{Z}$ score of voxels both inside and outside of the template, and then selecting the component maximizing the inside-outside difference. This process was completed for the following networks of interest: the DMN, EAN, and MVN, the latter of which is a control network with less direct association with the chronic pain state. Characteristically, the DMN includes the inferior parietal lobule (IPL) (Brodmann area 40 [BA40], BA39), the posterior cingulate cortex (PCC) (BA30, BA23, $\mathrm{BA} 31)$ and precuneus (BA7), areas of the medial frontal gyri (BA8, BA9, BA10, BA47), the hippocampal formation, and the lateral temporal cortex (BA21) (32). The EAN is typically split, by ICA, into a right and a left lateralized network, and includes the dorsolateral prefrontal cortex (dlPFC), comprising (roughly) the frontal eye fields (BA4, BA6, BA8) and posterior parietal regions overlapping the superior parietal lobule (BA7) and intraparietal sulcus (iPS) (BA7, BA40) $(14,18)$. The MVN primarily includes the primary visual cortex (VI) in the calcarine sulcus and medial parastriate regions, including the lingual gyrus (BA17, BA18) (14).

In the next stage of the dual-regression approach, the spatial IC maps identified from the population data were used as a spatial regressor in a generalized linear model (GLM) of the subject's resting-state FMRI data. This model was then used to find the subject-specific temporal dynamics within the
25 ICNs defined above. The time series for each component were then variance normalized (by subtracting the mean and dividing by the SD) and used as a temporal regressor in a GLM of the subject's resting-state FMRI data. In order to limit any residual shared variance with non-neuronal (e.g., cardiorespiratory physiologic) processes, this GLM also included temporal regressors from the white matter and ventricular regions (similar to that discussed in ref. 11), as well as regressors representing cardiac and respiratory variability, defined by convolving the heart rate time series and respiratory variations with appropriate cardiac and respiratory transfer functions, respectively, as suggested by Chang et al (33) and Birn et al (34). This allowed us to estimate subject-specific spatial maps for each component. These maps were then entered into our higher-level analyses (see below).

Group analyses were performed to evaluate differences in intrinsic brain connectivity between our 2 groups (patients with FM and healthy controls), as well as how this intrinsic connectivity shows covariance with spontaneous pain intensity in patients with FM. Group main-effects maps for both patients with FM and healthy controls, as well as between-group difference maps (calculated using unpaired $t$-tests for patients with FM versus healthy controls) were determined for each of our ICNs of interest (the DMN, EAN, and MVN). For both analyses, the FMRIB Local Analysis of Mixed Effects (FLAME) procedure was used, which involves Markov chain Monte Carlo sampling to estimate the true random-effects component of the between-subject mixedeffects variance (with degrees of freedom) at each voxel. For the results of the difference maps calculated by unpaired $t$-test, the threshold for significance was set at $P$ values less than 0.05 , cluster-corrected for multiple comparisons.

In order to more closely link intrinsic connectivity with the chronic pain state in patients with FM, an analysis of covariance (ANCOVA) was also performed for ICNs that demonstrated significant differences in intrinsic connectivity between patients with FM and healthy control subjects. The covariate of interest was the spontaneous level of pain (assessed as the pain intensity score) reported by the patients immediately prior to the resting-state FMRI scan. This pain score was adjusted for age, since age is known to influence ICN connectivity (35) and there was a significant correlation between spontaneous pain and age $(r=0.61)$. The ANCOVA was performed using a mixed-effects model, and the threshold for significance was set at $P$ values less than 0.05 , clustercorrected for multiple comparisons.

\section{RESULTS}

FMRI and clinical findings. Resting-state FMRI data were collected from all 36 female subjects in the study. In addition, clinical data on pain intensity at the time of the scan (self-reported pain score on a $0-10-\mathrm{mm}$ visual analog scale) were also collected from the patients with FM, and pain scores ranged from 0.0 to 8.1 (mean $\pm \mathrm{SD} 4.8 \pm 2.4$ ). As part of our dual-regression probabilistic ICA approach, we identified 25 ICs in the temporally concatenated 4-D population data set, from which the DMN, EAN, and MVN were robustly defined 


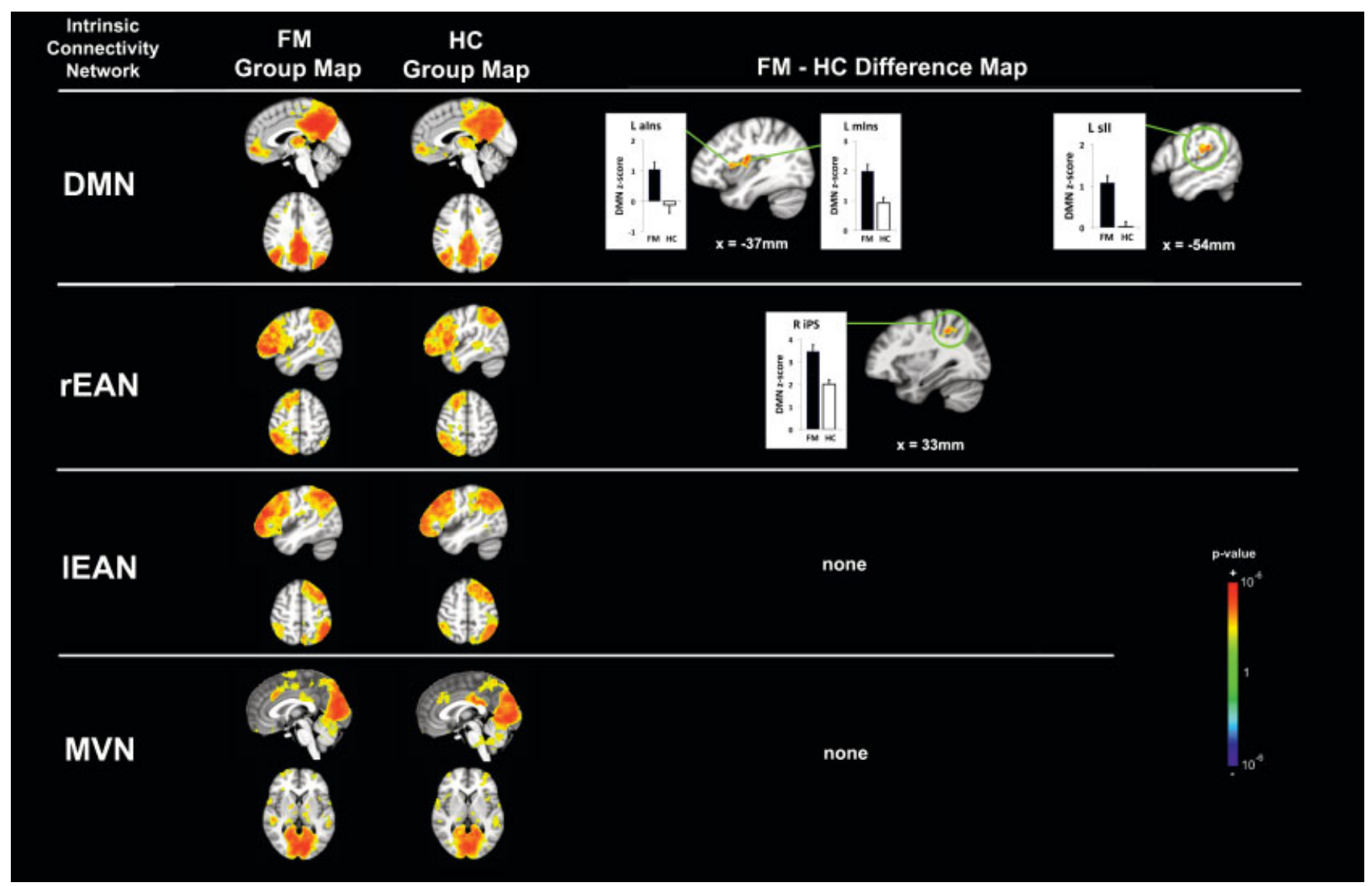

Figure 1. Within-group and between-group difference maps of intrinsic connectivity networks (ICNs) as assessed on functional magnetic resonance imaging. Group maps for healthy controls (HC) and patients with fibromyalgia (FM) (left) demonstrate the expected anatomic scope of the canonical default mode network (DMN), executive attention network (split into a right [rEAN] and left [IEAN] lateralized network), and medial visual network (MVN) for both groups. Difference maps contrasting patients with FM and healthy controls (right) demonstrate that patients with FM have greater intrinsic DMN connectivity to several brain regions that are outside of the DMN but are known to process evoked pain (the left anterior insula [L ains] and left middle insula [L mins]), as well as greater rEAN connectivity within this ICN (to the intraparietal sulcus [R iPS]). Bars show the mean and SD Z scores for functional connectivity in each group. L SII = left secondary somatosensory cortex.

(see group maps in Figure 1). As typically noted in past studies (14,36), the EAN was split into 2 lateralized networks, the right EAN and the left EAN.

Intrinsic brain connectivity in patients with FM compared with healthy controls. Of the ICNs evaluated, intrinsic connectivity within the DMN and right EAN demonstrated significant differences between patients with FM and healthy controls. Notably, in the DMN, the regional differences between the 2 groups were uniformly driven by greater positive DMN connectivity among patients with FM as compared with healthy controls (Table 2 and Figure 1). Patients with FM demonstrated greater intrinsic DMN connectivity to brain regions outside of the classic boundaries of the DMN, namely, the left anterior, middle, and posterior insula and the left secondary somatosensory cortex. No brain regions showed a greater correlation with the
DMN in healthy control subjects as compared with patients with FM.

Connectivity differences between patients with FM and healthy controls were also noted in the right EAN (Table 2 and Figure 1). Specifically, patients with FM demonstrated greater intranetwork connectivity within the right iPS. No brain regions showed a greater correlation with the right EAN in healthy control subjects as compared with patients with FM. Moreover, no differences between patients with FM and healthy controls were found in the left EAN or the MVN (our control network) (Figure 1).

Covariation between intrinsic connectivity and spontaneous pain. For analyses of covariation, ANCOVA was performed, with subjective pain intensity at the time of the scan serving as the covariate of interest. This analysis more closely links intrinsic brain connec- 
Table 2. Difference maps for comparison of intrinsic connectivity between patients with FM and healthy controls*

\begin{tabular}{|c|c|c|c|c|c|c|c|c|}
\hline & \multirow[b]{2}{*}{ Side } & \multirow[b]{2}{*}{ Size of area, $\mathrm{mm}^{2}$} & \multicolumn{3}{|c|}{ Location } & \multirow{2}{*}{$\begin{array}{l}\text { Between- } \\
\text { group } \\
\text { Z score }\end{array}$} & \multicolumn{2}{|c|}{$\mathrm{Z}$ score by group $\dagger$} \\
\hline & & & $\mathrm{X}$ & $\mathrm{Y}$ & $\mathrm{Z}$ & & Patients with FM & Healthy controls \\
\hline \multicolumn{9}{|l|}{ DMN } \\
\hline Anterior insula & Left & 552 & -34 & 6 & 10 & 3.33 & $1.04 \pm 1.05$ & $-0.12 \pm 1.15$ \\
\hline Middle insula & Left & 848 & -36 & -8 & 18 & 4.08 & $1.98 \pm 0.96$ & $0.92 \pm 0.81$ \\
\hline Posterior insula & Left & 704 & -46 & -30 & 22 & 3.25 & $1.80 \pm 0.95$ & $0.75 \pm 0.93$ \\
\hline SII & Left & 1,480 & -52 & -34 & 28 & 3.88 & $1.07 \pm 0.72$ & $0.02 \pm 0.53$ \\
\hline $\begin{array}{l}\text { Right EAN, } \\
\text { intraparietal sulcus }\end{array}$ & Right & 1,032 & 30 & -44 & 44 & 3.59 & $3.46 \pm 1.28$ & $2.00 \pm 0.90$ \\
\hline
\end{tabular}

* No differences between groups were observed in the left executive attention network (EAN) or the control medial visual network. FM = fibromyalgia; DMN = default mode network; SII = secondary somatosensory cortex.

$\dagger$ Values are the mean $\pm \mathrm{SD}$ within-group $\mathrm{Z}$ scores of intrinsic connectivity.

tivity to the chronic pain state. In the DMN, ANCOVA analysis demonstrated that greater spontaneous pain at the time of the FMRI scan was associated with greater DMN connectivity to the right anterior and right middle insula (Table 3 and Figure 2). A positive covariation with the intensity of spontaneous pain was also noted in the dlPFC, cerebellum, and subgenual anterior cingulate cortex.

In the right EAN, greater spontaneous pain correlated with greater intrinsic connectivity to the right anterior and left middle and posterior insula (Table 3 and Figure 3). A positive correlation with greater spontaneous pain was also noted between the right EAN and putamen, while a negative correlation (greater right EAN connectivity in relation to lower pain levels) was noted for the hippocampus, periaqueductal gray (PAG), nucleus cuneiformis, and pontine raphe.
In order to test for the influence of depression on any of our pain-related results, we also evaluated whether patients with FM classified as having a high level of depression (according to the number of symptoms of depression) had greater ICN connectivity to any of the brain regions implicated above. Based on our criteria, 7 patients had a high level of depression, while the remaining 11 patients were classified as having a low level of depression. We found no significant differences (all $P>0.2$ ) between these $2 \mathrm{FM}$ patient subpopulations (results not shown) in terms of ICN connectivity to regions of interest, as noted both in the FM-healthy control difference maps and in the ANCOVA results.

\section{DISCUSSION}

In this study, we present the first direct evidence of linkage between elevated intrinsic brain connectivity

Table 3. Covariation between intrinsic connectivity and spontaneous pain*

\begin{tabular}{|c|c|c|c|c|c|c|c|c|}
\hline & \multirow[b]{2}{*}{ Side } & \multirow{2}{*}{$\begin{array}{c}\text { Size of } \\
\text { area, } \\
\mathrm{mm}^{2}\end{array}$} & \multicolumn{3}{|c|}{ Location } & \multirow{2}{*}{$\begin{array}{c}\mathrm{Z} \\
\text { score }\end{array}$} & \multicolumn{2}{|c|}{$\mathrm{Z}$ score by pain intensity $\dagger$} \\
\hline & & & $X$ & $\mathrm{Y}$ & $\mathrm{Z}$ & & Low pain & High pain \\
\hline \multicolumn{9}{|l|}{$\mathrm{DMN}$} \\
\hline Anterior insula & Right & 464 & 42 & 14 & 8 & 3.29 & $-1.72 \pm 0.81$ & $0.55 \pm 1.32$ \\
\hline Middle insula & Right & 688 & 38 & -10 & 6 & 3.43 & $0.80 \pm 0.47$ & $3.06 \pm 0.49$ \\
\hline dlPFC & Right & 736 & 60 & -14 & 34 & 3.09 & $-0.84 \pm 1.44$ & $1.07 \pm 0.99$ \\
\hline Cerebellum & Left & 808 & -22 & -74 & -40 & 3.86 & $-1.37 \pm 0.49$ & $0.33 \pm 0.71$ \\
\hline sgACC & Right & 248 & 6 & 26 & -12 & 3.75 & $-1.11 \pm 0.98$ & $1.12 \pm 0.30$ \\
\hline \multicolumn{9}{|l|}{ Right EAN } \\
\hline Anterior insula & Right & 816 & 28 & 26 & -6 & 3.78 & $0.57 \pm 0.48$ & $2.72 \pm 0.95$ \\
\hline Middle insula & Left & 400 & -36 & -8 & 10 & 2.88 & $-1.28 \pm 1.11$ & $1.14 \pm 0.89$ \\
\hline Posterior insula & Left & 336 & -32 & -20 & 14 & 2.79 & $-1.17 \pm 1.37$ & $0.24 \pm 0.51$ \\
\hline Hippocampus & Right & 160 & 18 & -20 & -18 & -3.07 & $1.39 \pm 0.86$ & $-0.10 \pm 0.48$ \\
\hline Putamen & Right & 408 & 24 & 16 & -6 & 3.12 & $-0.02 \pm 0.47$ & $1.80 \pm 1.11$ \\
\hline PAG & Left & 256 & -2 & -26 & -10 & -3.58 & $1.45 \pm 0.91$ & $-0.52 \pm 1.68$ \\
\hline Nucleus cuneiformis & Right & 320 & 12 & -26 & -16 & -3.40 & $1.07 \pm 1.32$ & $-0.63 \pm 0.70$ \\
\hline Pontine raphe & Left & 496 & -2 & -30 & -28 & -4.15 & $1.55 \pm 0.56$ & $0.02 \pm 0.63$ \\
\hline
\end{tabular}

${ }^{*} \mathrm{DMN}=$ default mode network; dlPFC $=$ dorsolateral prefrontal cortex; sgACC $=$ subgenual anterior cingulated cortex; EAN $=$ executive attention network; PAG = periaqueductal gray.

$\dagger$ Values are the mean \pm SD Z scores for intrinsic connectivity in the 4 patients with fibromyalgia reporting the highest and lowest spontaneous pain intensities (on a visual analog scale of $0-10 \mathrm{~mm}$ ) at the time of the scan. 


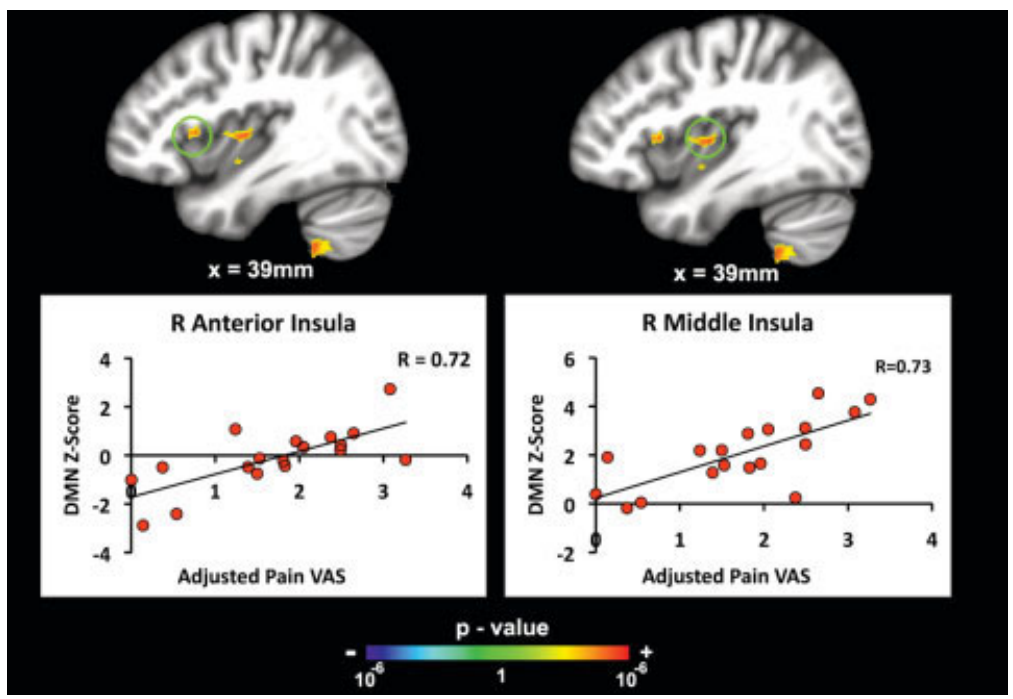

Figure 2. Covariation between default mode network (DMN) connectivity and age-adjusted spontaneous pain as assessed on functional magnetic resonance imaging (top) and as DMN Z scores according to visual analog scale (VAS) scores of pain intensity. Greater spontaneous pain intensity correlated with linearly increasing intrinsic DMN connectivity to the right $(\mathrm{R})$ anterior and right middle insula. Circles represent individual patients with fibromyalgia. Representative images are shown. Color figure can be viewed in the online issue, which is available at http://www. arthritisrheum.org.

and spontaneous pain intensity in patients with FM. We applied ICA to resting-state FMRI data and found that patients with FM had greater connectivity within the right EAN and between the DMN and the insular cortex, a brain region linked to evoked pain processing. Furthermore, our data directly link ratings of self-

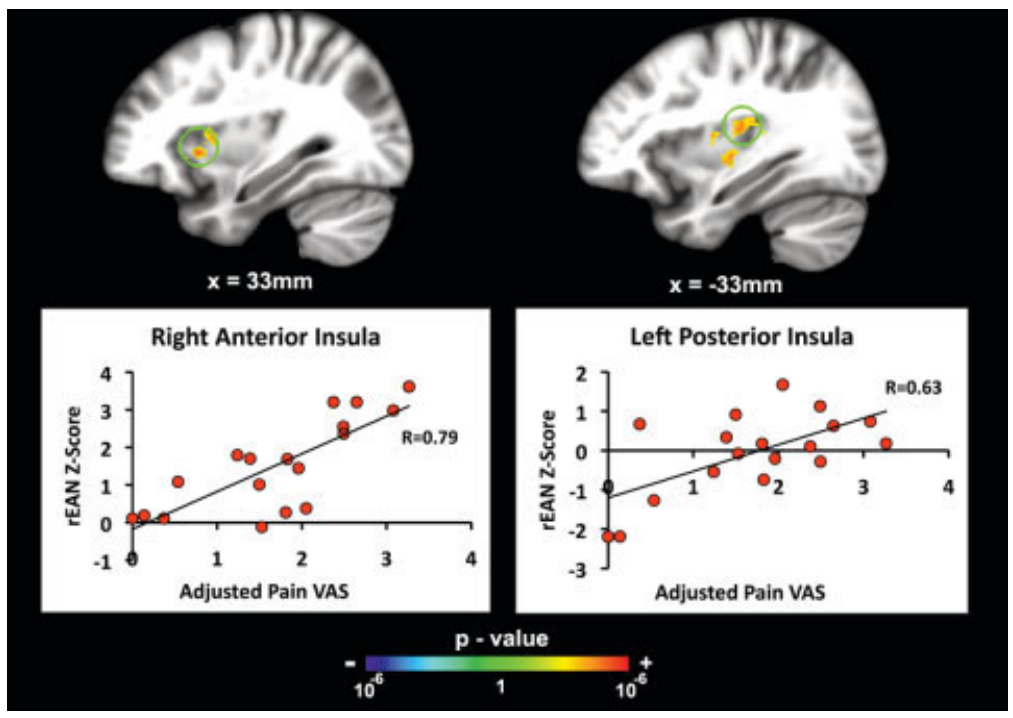

Figure 3. Covariation between right executive attention network (rEAN) connectivity and age-adjusted spontaneous pain as assessed on functional magnetic resonance imaging (top) and as DMN Z scores according to visual analog scale (VAS) scores of pain intensity. Greater spontaneous pain intensity correlated with linearly increasing intrinsic rEAN connectivity to the right anterior insula and left posterior insula. Circles represent individual patients with fibromyalgia. Representative images are shown. Color figure can be viewed in the online issue, which is available at http://www.arthritisrheum.org. 
reported spontaneous pain at the time of the scan to the degree of both right EAN and DMN connectivity to the insula. Our findings have implications for a better understanding of the underlying brain mechanisms of endogenous clinical pain in FM, potentially pointing toward markers of disease progression. More broadly, these findings have implications for clarifying how subjective experiences such as pain arise from a complex interplay among multiple brain networks.

Our results strongly implicate the insular cortex as being a key node in the elevated intrinsic connectivity in patients with FM. Patients demonstrated greater DMN connectivity to the left anterior, middle, and posterior insula. Furthermore, patients with FM who reported experiencing greater spontaneous pain displayed increased intrinsic connectivity between both the DMN and right EAN and insular cortex. Many studies have found insular involvement in the multidimensional (sensory, affective, cognitive) pain state. The insula comprise one of the most commonly activated brain regions in neuroimaging studies of acute experimental pain (37). The posterior insula has been associated with sensory intensity encoding (38), while the anterior insula may be more strongly related to affective dimensions of pain, such as anticipatory anxiety related to pain (39). However, the insula do not simply process pain signals; rather, they have also been implicated in multiple associative processes related to both interoceptive (40) and exteroceptive (18) salience. In fact, it has been hypothesized that the insula integrate subcortical homeostatic information, such as that arising from a pain state (41), into a higher-order cognitive and affective conscious state of awareness (42). Our data support this view, in that ratings of spontaneous pain were correlated with increased insular connectivity in brain networks (the DMN and EAN) known to support cognition.

Intrinsic connectivity between the posterior insula and DMN areas such as the PCC has been shown to exist even in healthy subjects (43). Substantial positive DMN connectivity to the posterior insula was also noted in our analysis, both in patients with FM and in healthy controls. This suggests that existing links between the DMN and insula may be hyperactive in patients with FM. This hyperactive connectivity has been suggested by proton magnetic resonance spectroscopy studies in patients with FM in whom posterior insula glutamate levels appeared to be elevated (44). Future studies should also explore increased glutamate concentrations within the DMN, since we found increased intrinsic DMN connectivity in patients with FM. In addition, greater DMNinsula connectivity in the resting state could lead to an altered DMN response to external sensory input in patients with FM, an anomaly that was previously observed in patients with chronic low back pain responding to cognitive/visual tasks (45).

It is also interesting to speculate that pain-related reorganization of ICNs associated with working memory and attention (i.e., the EAN) provides a potential neurobiologic mechanism for the known cognitive deficits in patients with FM (for review of FM-related dyscognition, see ref. 20). Perhaps not coincidentally, these deficits appear predominantly in the cognitive areas of working memory and attention, and are particularly exacerbated with increasing distraction. Of particular note, cognitive deficits in patients with FM are correlated more with their level of pain than with psychiatric comorbidities (e.g., depression, anxiety, or sleep disruption), as shown in a recent study by Dick et al (46). Our results indicate that connectivity of the insula with the right EAN in patients with FM increases with increasing levels of spontaneous pain. Interestingly, PAG-right EAN connectivity decreases with increasing pain, consistent with the hypothesis that cognitive controls for descending inhibition (antinociception) might modulate pain levels in patients with FM. Our results suggest that the insula, which are intimately involved with multidimensional aspects of pain processing, become more hyperconnected to the right EAN as pain levels increase in patients with FM, thus diverting resources away from normal right EAN functioning and thereby leading to deficits in working memory and attention.

We found altered intrinsic connectivity in the right, but not left, EAN. The ICA approach commonly separates the EAN into a right and a left lateralized network (14,36). Laterality in working memory and attention tasks has been noted in previous neuroimaging studies (47), and lesions of the iPS typically result in spatial attention deficits when localized to the right side (48). This laterality corroborates our suggestion that altered intrinsic right EAN connectivity may play a role in dyscognition in patients with FM. Future studies should explore the significance of right, versus left, EAN connectivity in patients with FM.

Another interesting finding was that the differences in DMN and EAN connectivity between patients with FM and healthy controls were driven entirely by the alterations observed in the FM group, and not in the healthy controls. Although this result could indicate that the observed differences were either the consequence of or the cause of lingering chronic pain, it certainly supports the growing body of evidence that FM, and likely other chronic pain syndromes, are accompanied by altered brain neurophysiology. Moreover, this result was qualitatively similar to that reported recently in a study 
showing altered ICN connectivity in patients with other forms of chronic pain (49). Specifically, Cauda et al found an enhanced resting-state DMN connectivity within DMN component regions (precuneus, IPL), sensorimotor regions (bilateral thalamus and insula), and cognitive/evaluative pain-modulatory regions (dlPFC) (49). However, our findings substantially add to this intergroup comparison, since we found that the degree of DMN connectivity was associated with the level of state-specific self-reported pain at the time of the scan, which is an important finding because it specifically links intrinsic functional connectivity to the chronic pain state. The specificity of our results was buttressed by the fact that a control brain network, the MVN (not previously implicated in FM pathology), did not display altered intrinsic connectivity. Thus, our findings of altered brain connectivity do not appear to be widespread, but are localized to the ICNs of the DMN and right EAN.

Other neuroimaging approaches have also attempted to evaluate the brain correlates of spontaneous clinical pain. Positron emission tomography with opioidbinding agents has shown that patients with FM have decreased binding potential within the nucleus accumbens, and this is correlated with increasing spontaneous pain (50). A proton magnetic resonance spectroscopy study found that reductions in resting glutamate concentrations in the posterior insula are associated with reductions in the level of self-reported clinical pain in patients with FM (51). Finally, in a study that used continuous, online patient self-report of spontaneous pain fluctuation to guide the statistical analyses of the FMRI data, the results indicated that the FMRI signal in the medial prefrontal cortex (MPFC) is correlated with spontaneous pain intensity (1). Our results add to this growing literature on neural correlates of chronic pain. The MPFC is a cardinal node of the DMN, and our results point to increased intrinsic DMN connectivity to the insula, with increasing connectivity directly correlated with increasing levels of spontaneous clinical pain in patients with FM.

There are a number of limitations to our study that should be discussed. First, our results were derived strictly from patients with FM and may not be generalizable to other chronic pain states, a possibility that we are currently evaluating. However, qualitatively similar findings have been observed in patients with neuropathic pain (49), and therefore our results likely do have some generalizability. Furthermore, all of our participants were female, leaving open the possibility that FM pathophysiology may, in fact, be different in male patients. Finally, some of our patients with FM were taking medications during the time of the scan (details available from the corresponding author upon request), and thus some findings may have been influenced by pharmacologic modulation of neural connectivity. For ethical reasons, subjects were not asked to titrate down their existing pain medications for this study. However, none of our patients were taking opioid medications, and since the effects of other medications (e.g., selective serotonin reuptake inhibitors or serotoninnorepinephrine reuptake inhibitors) on intrinsic brain connectivity are unknown, they should be explored in future studies. Larger studies, which could group participants by medication usage, can also address this issue.

In conclusion, the results of this study provide direct evidence of disrupted intrinsic connectivity within multiple brain networks in patients with FM. These findings are in agreement with other brain imaging results that have indicated that individuals with FM have altered brain function $(5,6,44,50)$. Emerging evidence suggests that pain associated with FM may be mediated by central nervous system hyperexcitability rather than peripheral dysfunction. Our results clearly show that individuals with FM have greater connectivity between multiple brain networks and the insular cortex, which is a brain region previously linked with evoked pain processing and hyperexcitability in FM. Our data also demonstrate that intrinsic connectivity to the insula is directly associated with increasing spontaneous pain. Thus, our approach represents a novel step forward in finding the neural correlates of spontaneous clinical pain.

\section{ACKNOWLEDGMENTS}

We would like to thank the National Center for Complementary and Alternative Medicine, the National Center for Research Resources, the Mental Illness and Neuroscience Discovery Institute, the Institute of Information Technology Advancement in Korea, the US Department of Defense, and the Dana Foundation for funding support. We also acknowledge support from Pfizer, Inc.

\section{AUTHOR CONTRIBUTIONS}

All authors were involved in drafting the article or revising it critically for important intellectual content, and all authors approved the final version to be published. Dr. Napadow had full access to all of the data in the study and takes responsibility for the integrity of the data and the accuracy of the data analysis.

Study conception and design. Napadow, Clauw, Harris.

Acquisition of data. Napadow, Clauw, Harris.

Analysis and interpretation of data. Napadow, LaCount, Park, AsSanie, Clauw, Harris.

\section{REFERENCES}

1. Baliki MN, Chialvo DR, Geha PY, Levy RM, Harden RN, Parrish $\mathrm{TB}$, et al. Chronic pain and the emotional brain: specific brain 
activity associated with spontaneous fluctuations of intensity of chronic back pain. J Neurosci 2006;26:12165-73.

2. Tracey I. Imaging pain. Br J Anaesth 2008;101:32-9.

3. Harris RE, Williams DA, McLean SA, Sen A, Hufford M, Gendreau RM, et al. Characterization and consequences of pain variability in individuals with fibromyalgia. Arthritis Rheum 2005; 52:3670-4.

4. Clauw D, Williams D. Fibromyalgia. In: Mayer E, Bushnell M, editors. Functional pain syndromes: presentation and pathophysiology. Seattle: IASP Press; 2009. p. 580.

5. Cook DB, Lange G, Ciccone DS, Liu WC, Steffener J, Natelson $\mathrm{BH}$. Functional imaging of pain in patients with primary fibromyalgia. J Rheumatol 2004;31:364-78.

6. Gracely RH, Petzke F, Wolf JM, Clauw DJ. Functional magnetic resonance imaging evidence of augmented pain processing in fibromyalgia. Arthritis Rheum 2002;46:1333-43.

7. Harris RE, Gracely RH, McLean SA, Williams DA, Giesecke T, Petzke F, et al. Comparison of clinical and evoked pain measures in fibromyalgia. J Pain 2006;7:521-7.

8. Raichle ME, Mintun MA. Brain work and brain imaging. Annu Rev Neurosci 2006;29:449-76.

9. Fox MD, Raichle ME. Spontaneous fluctuations in brain activity observed with functional magnetic resonance imaging. Nat Rev Neurosci 2007;8:700-11.

10. Van den Heuvel MP, Mandl RC, Kahn RS, Hulshoff Pol HE. Functionally linked resting-state networks reflect the underlying structural connectivity architecture of the human brain. Hum Brain Mapp 2009;30:3127-41.

11. Krienen FM, Buckner RL. Segregated fronto-cerebellar circuits revealed by intrinsic functional connectivity. Cereb Cortex 2009; 19:2485-97.

12. Raichle ME. A paradigm shift in functional brain imaging. J Neurosci 2009;29:12729-34.

13. Foss JM, Apkarian AV, Chialvo DR. Dynamics of pain: fractal dimension of temporal variability of spontaneous pain differentiates between pain states. J Neurophysiol 2006;95:730-6.

14. Beckmann CF, DeLuca M, Devlin JT, Smith SM. Investigations into resting-state connectivity using independent component analysis. Philos Trans R Soc Lond B Biol Sci 2005;360:1001-13.

15. Buckner RL, Vincent JL. Unrest at rest: default activity and spontaneous network correlations. Neuroimage 2007;37:1091-6.

16. Seminowicz DA, Davis KD. Pain enhances functional connectivity of a brain network evoked by performance of a cognitive task. J Neurophysiol 2007;97:3651-9.

17. Baliki M, Geha P, Apkarian A, Chialvo D. Beyond feeling: chronic pain hurts the brain, disrupting the default-mode network dynamics. J Neurosci 2008;28:1398-403.

18. Seeley WW, Menon V, Schatzberg AF, Keller J, Glover GH, Kenna $\mathrm{H}$, et al. Dissociable intrinsic connectivity networks for salience processing and executive control. J Neurosci 2007;27: 2349-56.

19. Corbetta M, Shulman GL. Control of goal-directed and stimulusdriven attention in the brain. Nat Rev Neurosci 2002;3:201-15.

20. Glass JM. Review of cognitive dysfunction in fibromyalgia: a convergence on working memory and attentional control impairments. Rheum Dis Clin North Am 2009;35:299-311.

21. Dhond RP, Yeh C, Park K, Kettner N, Napadow V. Acupuncture modulates resting state connectivity in default and sensorimotor brain networks. Pain 2008;136:407-18.

22. Newton AT, Morgan VL, Gore JC. Task demand modulation of steady-state functional connectivity to primary motor cortex. Hum Brain Mapp 2007;28:663-72.

23. Wolfe F, Smythe HA, Yunus MB, Bennett RM, Bombardier C, Goldenberg DL, et al. The American College of Rheumatology 1990 criteria for the classification of fibromyalgia: report of the Multicenter Criteria Committee. Arthritis Rheum 1990;33:160-72.

24. Zigmond AS, Snaith RP. The Hospital Anxiety and Depression Scale. Acta Psychiatr Scand 1983;67:361-70.
25. Radloff L. The CES-D Scale: a self-report depression scale for research in the general population. Appl Psychol Meas 1977;1: 385-401.

26. Birn RM, Diamond JB, Smith MA, Bandettini PA. Separating respiratory-variation-related fluctuations from neuronal-activityrelated fluctuations in fMRI. Neuroimage 2006;31:1536-48.

27. Chang C, Glover GH. Effects of model-based physiological noise correction on default mode network anti-correlations and correlations. Neuroimage 2009;47:1448-59.

28. Kiviniemi VJ, Haanpaa H, Kantola JH, Jauhiainen J, Vainionpaa $\mathrm{V}$, Alahuhta S, et al. Midazolam sedation increases fluctuation and synchrony of the resting brain BOLD signal. Magn Reson Imaging 2005;23:531-7.

29. Larson-Prior LJ, Zempel JM, Nolan TS, Prior FW, Snyder AZ, Raichle ME. Cortical network functional connectivity in the descent to sleep. Proc Natl Acad Sci U S A 2009;106:4489-94.

30. Filippini N, MacIntosh BJ, Hough MG, Goodwin GM, Frisoni GB, Smith SM, et al. Distinct patterns of brain activity in young carriers of the APOE- $\epsilon 4$ allele. Proc Natl Acad Sci U S A 2009;106:7209-14.

31. Zuo XN, Kelly C, Adelstein JS, Klein DF, Castellanos FX, Milham MP. Reliable intrinsic connectivity networks: test-retest evaluation using ICA and dual regression approach. Neuroimage 2010;49: 2163-77.

32. Buckner RL, Andrews-Hanna JR, Schacter DL. The brain's default network: anatomy, function, and relevance to disease. Ann N Y Acad Sci 2008;1124:1-38.

33. Chang C, Cunningham JP, Glover GH. Influence of heart rate on the BOLD signal: the cardiac response function. Neuroimage 2009;44:857-69.

34. Birn RM, Smith MA, Jones TB, Bandettini PA. The respiration response function: the temporal dynamics of fMRI signal fluctuations related to changes in respiration. Neuroimage 2008;40: 644-54.

35. Damoiseaux JS, Beckmann CF, Arigita EJ, Barkhof F, Scheltens $\mathrm{P}$, Stam CJ, et al. Reduced resting-state brain activity in the "default network" in normal aging. Cereb Cortex 2008;18: $1856-64$.

36. Damoiseaux JS, Rombouts SA, Barkhof F, Scheltens P, Stam CJ, Smith SM, et al. Consistent resting-state networks across healthy subjects. Proc Natl Acad Sci U S A 2006;103:13848-53.

37. Apkarian AV, Bushnell MC, Treede RD, Zubieta JK. Human brain mechanisms of pain perception and regulation in health and disease. Eur J Pain 2005;9:463-84.

38. Craig AD, Chen K, Bandy D, Reiman EM. Thermosensory activation of insular cortex. Nat Neurosci 2000;3:184-90.

39. Ploghaus A, Tracey I, Gati JS, Clare S, Menon RS, Matthews PM, et al. Dissociating pain from its anticipation in the human brain. Science 1999;284:1979-81.

40. Craig AD. How do you feel? Interoception: the sense of the physiological condition of the body. Nat Rev Neurosci 2002;3: 655-66.

41. Craig AD. A new view of pain as a homeostatic emotion. Trends Neurosci 2003;26:303-7.

42. Craig AD. How do you feel-now? The anterior insula and human awareness. Nat Rev Neurosci 2009;10:59-70.

43. Taylor KS, Seminowicz DA, Davis KD. Two systems of resting state connectivity between the insula and cingulate cortex. Hum Brain Mapp 2009;30:2731-45.

44. Harris RE, Sundgren PC, Craig AD, Kirshenbaum E, Sen A, Napadow V, et al. Elevated insular glutamate in fibromyalgia is associated with experimental pain. Arthritis Rheum 2009;60: 3146-52.

45. Baliki MN, Geha PY, Apkarian AV, Chialvo DR. Beyond feeling: chronic pain hurts the brain, disrupting the default-mode network dynamics. J Neurosci 2008;28:1398-403.

46. Dick BD, Verrier MJ, Harker KT, Rashiq S. Disruption of 
cognitive function in fibromyalgia syndrome. Pain 2008;139:610-6.

47. Sandrini M, Rossini PM, Miniussi C. Lateralized contribution of prefrontal cortex in controlling task-irrelevant information during verbal and spatial working memory tasks: rTMS evidence. Neuropsychologia 2008;46:2056-63.

48. Vandenberghe R, Gillebert CR. Parcellation of parietal cortex: convergence between lesion-symptom mapping and mapping of the intact functioning brain. Behav Brain Res 2009;199:171-82.

49. Cauda F, Sacco K, Duca S, Cocito D, D’Agata F, Geminiani GC, et al. Altered resting state in diabetic neuropathic pain. PLoS ONE 2009; 4:e4542.

50. Harris RE, Clauw DJ, Scott DJ, McLean SA, Gracely RH, Zubieta JK. Decreased central $\mu$-opioid receptor availability in fibromyalgia. J Neurosci 2007;27:10000-6.

51. Harris RE, Sundgren PC, Pang Y, Hsu M, Petrou M, Kim SH, et al. Dynamic levels of glutamate within the insula are associated with improvements in multiple pain domains in fibromyalgia. Arthritis Rheum 2008;58:903-7.

In the article by Kavanaugh et al in the April 2009 issue of Arthritis \& Rheumatism (pages 976-986), there were several errors in Tables 1 and 2, resulting from an unintentional systematic programming error pertaining to CRP units used in the DAS28-CRP calculations. In Table 1, the mean \pm SD DAS-CRP values in the placebo group, the golimumab $50 \mathrm{mg}$ group, and the golimumab $100 \mathrm{mg}$ group, respectively, should have been reported as $4.9 \pm 1.0,5.0 \pm 1.1$, and $4.9 \pm 1.1$. In Table 2, the mean \pm SD change in the DAS28-CRP at week 14 should have been $-0.19 \pm 0.81,-1.49 \pm 1.19$, and $-1.41 \pm 1.19$, respectively, and the mean \pm SD change in the DAS28-CRP at week 24 should have been $-0.12 \pm 1.01,-1.53 \pm 1.38$, and $-1.67 \pm 1.13$, respectively. Also in Table 2, the number (\%) of patients in the placebo group, the golimumab 50 mg group, and the golimumab $100 \mathrm{mg}$ group, respectively, achieving EULAR response at week 14 should have been reported as 27 (24), 100 (69), and 100 (69), and the number (\%) achieving EULAR response at week 24 should have been 25 (22), 95 (65), and 117 (80) (no changes in $P$ values). The errors did not affect the significance of the DAS-CRP analyses or the interpretation or conclusions of the study.

DOI 10.1002/art.27674

In the review by Monach et al in the January 2010 issue of Arthritis \& Rheumatism (pages 9-21), there were several errors in the text of the fourth paragraph on page 15. The paragraph should have read as follows: "The recommended dosing of IV mesna is a total dose equal to $60 \%$ (weight/weight) of the total cyclophosphamide dose, in the form of 3 equal doses of mesna ( $20 \%$ each of the total dose), with the first dose administered 15-30 minutes prior to cyclophosphamide and the others administered 4 hours and 8 hours following cyclophosphamide (67). When mesna is given orally, the dose should be equal to $40 \%$ of the cyclophosphamide dose (oral or IV), based on the $50 \%$ oral bioavailability of mesna. For convenience, a combination of IV and oral doses can be given: an initial IV dose (equal to $20 \%$ of the cyclophosphamide dose) followed by 2 oral doses (each equal to $40 \%$ of the cyclophosphamide dose). If the first dose of mesna is administered orally, it should be given 2 hours before cyclophosphamide (oral or IV), but the second and third oral doses can still be given 4 hours and 8 hours after cyclophosphamide, as with IV mesna dosing."

We regret the errors. 